\title{
Unknown Primary Tumor (Except for EBV- Related and HPV-Related Tumors) and Metastatic Cervical Adenopathy by AJCC v8 Stage
}

National Cancer Institute

\section{Source}

National Cancer Institute. Unknown Primary T umor (Except for EBV-Related and HPV-

Related Tumors) and Metastatic Cervical Adenopathy by A/CC v8 Stage. NCI Thesaurus. Code C132676.

A term that refers to the staging of unknown primary tumor (except for EBV-related and HPV-related tumors) and metastatic cervical adenopathy according to the American Joint Committee on Cancer, 8th edition. 